\title{
Stability Analysis of Markovian Jump Systems with Multiple Delay Components and Polytopic Uncertainties
}

\author{
Qing Wang • Baozhu Du • James Lam • \\ Michael Z.Q. Chen
}

Received: 19 August 2010 / Revised: 22 February 2011 / Published online: 26 March 2011

(C) The Author(s) 2011. This article is published with open access at Springerlink.com

\begin{abstract}
This paper investigates the stability problem of Markovian jump systems with multiple delay components and polytopic uncertainties. A new LyapunovKrasovskii functional is used for the stability analysis of Markovian jump systems with or without polytopic uncertainties. Two numerical examples are provided to demonstrate the applicability of the proposed approach.
\end{abstract}

Keywords Delay $\cdot$ Markovian jump system $\cdot$ Stability $\cdot$ Polytopic uncertainty

This work was supported by National Natural Science Foundation of China under Grant 60804032 and by the Hong Kong Research Grants Council under HKU 7137/09E.

Q. Wang $(\bowtie)$

Department of Electrical and Electronic Engineering, The University of Hong Kong, Pokfulam Road, Hong Kong, Hong Kong

e-mail: sunsanqingwang@gmail.com

Q. Wang

School of Information Science and Technology, Sun Yat-sen University, Guangzhou, 510275, China

B. Du

Department of Mathematics, Dalian Maritime University, 116026, Dalian, China

e-mail: dubaozhu@gmail.com

J. Lam · M.Z.Q. Chen

Department of Mechanical Engineering, The University of Hong Kong, Pokfulam Road, Hong Kong, Hong Kong

J. Lam

e-mail: james.lam@hku.hk

M.Z.Q. Chen

e-mail: mzqchen@hku.hk 


\section{Introduction}

Markovian jump linear systems are hybrid systems with many operation models and each model corresponds to a deterministic dynamic system. The switching amongst the system modes is governed by a Markov process. This class of systems is often used to model systems whose structures are subject to abrupt changes and their extensive applications have been applied to many physical systems such as manufacturing systems, aircraft control, target tracking, robotics, solar receiver control, power systems, and so on $[2,18]$. Considerable attention has recently been devoted to the study of Markovian jump linear systems such as controllability [16, 17], stability [5, 10, 37], $H_{\infty}$ control [2, 7, 19, 23, 28], $H_{2}$ control [9], $H_{\infty}$ filtering [11, 22, 26, 36, 39], guaranteed cost control [8], and model reduction [30, 42].

As time delay is very common in many physical, industrial and engineering systems [1, 24, 25], it is often introduced to model these systems; for example, multiple delays are required to model the RLC circuits with delayed elements [29], networked control systems [14, 20] and nonlinear stochastic systems [1]. However, since dynamic systems with time delays are of infinite dimensions, the control of these systems is fairly complicated and the desired performance of the closed-loop systems is difficult to achieve. Therefore, the presence of time delays substantially complicates the analytical and theoretical aspects of control system design. A survey of recent results developed to analyze the stability of delay systems is given in [38], which includes delay-independent and delay-dependent results. Recently, a new simplified and more efficient stability criterion for linear continuous system with multiple components has been used in [13] to improve the delay-dependent stability condition. As a result, the study of Markovian jump linear systems with time delay has received attention of many researchers in topics of stability [40], $H_{\infty}$ control [4, 31, 34], and filtering [12, 32, 33].

To accurately characterize the physical systems, description of the uncertainty in the system parameters is often needed. Two types of uncertainties, norm bounded uncertainties and polytopic ones, are commonly used [6]. For jump systems with norm bounded uncertainties, the stability and stabilization [5, 21, 35, 41], $H_{\infty}$ control [15], and robustness $H_{\infty}$ filtering [36] have been addressed. The robust stability and $H_{\infty}$ control of Markovian jump systems with constant delay and polytopic uncertainties is studied in [4].

In this paper, we are concerned with the robust stability of continuous-time Markovian jump systems with mode-dependent multiple time delays and time-varying polytopic uncertainties. The rest of the paper is organized as follows. Section 2 describes the continuous-time Markovian jump systems with mode-dependent multiple time delays and polytopic uncertainties, and presents preliminary results. The main results are given in Sect. 3, which includes the stability and robust stability of continuoustime Markovian jump system with mode-dependent multiple time delays without polytopic uncertainties or with polytopic uncertainties, respectively. Two numerical examples are used to illustrate the main results in Sect. 4, which is followed by the conclusion in Sect. 5.

Notation Throughout this paper, the notation $X>Y$ for real symmetric matrices $X$ and $Y$ means that the matrix $X-Y$ is positive definite. $\mathbb{R}^{n}$ is the set of all column 
vectors with $n$ real entries. $M^{T}$ represents the transpose of the matrix $M$. Identity matrices are invariably denoted by $I$ when their dimensions are obvious and otherwise denoted by $I_{n}$ to represent an $n \times n$ identity matrix, while zero matrices are invariably denoted by 0 . The notation $\operatorname{col}\{\cdot\}$ denotes a matrix column with blocks given by the matrices in $\{\cdot\}$. For a matrix $M \in \mathbb{R}^{n \times m}$ with rank $r$, the orthogonal complement is defined as a (possibly non-unique) $m \times(m-r)$ matrix such that $M M^{\perp}=0$ and $M^{\perp T} M^{\perp}>0$.

\section{Problem Formulation}

Given is a probability space $(\Omega, \mathcal{F}, \mathcal{P})$ where $\Omega$ is the sample space, $\mathcal{F}$ is the $\sigma$ algebra of subsets of the sample space and $\mathcal{P}$ is the probability measure defined on $\mathcal{F}$. On this probability space $(\Omega, \mathcal{F}, \mathcal{P})$, we consider the following class of continuoustime Markovian jump systems with mode-dependent multiple time delays and polytopic uncertainties:

$$
\begin{aligned}
\Sigma_{p}: \quad \dot{x}(t) & =A\left(r_{t}\right) x(t)+A_{d}\left(r_{t}\right) x\left(t-\sum_{k=1}^{r} d_{k}\left(r_{t}\right)\right), \\
x(t) & =\varphi(t), \quad t \in[-\mu, 0],
\end{aligned}
$$

where $x(t) \in \mathbb{R}^{n}$ is the state, $\left\{r_{t}\right\}$ is a continuous-time homogeneous Markov process with right-continuous trajectories and taking values in a finite set $\tilde{N}=\{1, \ldots, N\}$, and stationary transition probability matrix $\Pi \triangleq\left[\pi_{i j}\right]_{i, j \in \tilde{N}}$ is given by

$$
\operatorname{Pr}\left\{r_{t+h}=j \mid r_{t}=i\right\}= \begin{cases}\pi_{i j} h+o(h) & i \neq j \\ 1+\pi_{i i} h+o(h) & i=j\end{cases}
$$

where $h>0, \lim _{h \rightarrow 0}(o(h) / h)=0$, and $\pi_{i j} \geq 0$, for $i \neq j$, is the transition rate from mode $i$ at time $t$ to mode $j$ at time $t+h$, and

$$
\pi_{i i}=-\sum_{j=1, j \neq i}^{N} \pi_{i j}
$$

The $d_{k}\left(r_{t}\right), k=1, \ldots, r$, denote the time delay components in the state and are assumed to satisfy the following conditions:

$$
0<d_{k}\left(r_{t}\right)=d_{i k} \leq \bar{d}_{i k}<\infty, \quad r_{t}=i \in \tilde{N},
$$

and

$$
\mu=\max _{i \in \tilde{N}}\left\{\sum_{k=1}^{r} \bar{d}_{i k}\right\} .
$$

In (1), $\varphi(t)$ is a vector-valued initial continuous function defined on the interval $[-\mu, 0]$. When system $\Sigma_{d}$ is in mode $r_{t}, A\left(r_{t}\right)$ and $A_{d}\left(r_{t}\right)$ are matrix functions of $r_{t}$. 
For each possible value $r_{t}=i, i \in \tilde{N}$, the matrices associated with the $i$ th mode are unknown and assumed to belong to a convex compact set of polytopic type,

$$
\left[A(i) \quad A_{d}(i)\right] \in U_{p}(i),
$$

where

$$
U_{p}(i) \triangleq\left\{\sum_{l=1}^{q} \alpha_{l}(t)\left[\begin{array}{ll}
A_{i l} & A_{d i l}
\end{array}\right], \forall \alpha_{l}(t) \geq 0, \sum_{l=1}^{q} \alpha_{l}(t)=1\right\}
$$

with $A_{i l}$ and $A_{d i l}$ being given matrices with appropriate dimensions.

Definition 1 [4] System $\Sigma_{p}$ is said to be robust stochastically stable if for any $A(i)$ and $A_{d}(i) \in U_{p}(i)$, there exists a constant $U_{0}\left(\varphi(\cdot), r_{0}\right)$, which is dependent on the initial condition $\left(\varphi(\cdot), r_{0}\right)$ and satisfies $U_{0}(0,0)=0$, such that

$$
\mathbb{E}\left[\int_{0}^{\infty}\left\|x\left(t, \varphi(\cdot), r_{0}\right)\right\|^{2} d t\right] \leq U_{0}\left(\varphi(\cdot), r_{0}\right),
$$

where $\mathbb{E}[\cdot]$ denotes the expectation and $x\left(t, \varphi(\cdot), r_{0}\right)$ denotes the solution of system $\Sigma_{p}$ at time $t$ under the initial conditions $\varphi(\cdot)$ and $r_{0}$.

Lemma 1 [13] Let $Y \in \mathbb{R}^{n \times n}$ and the bidiagonal upper triangular block matrix be

$$
J_{K}(Y)=\left[\begin{array}{ccccc}
I_{n} & -Y & 0 & \cdots & 0 \\
0 & I_{n} & -Y & \cdots & 0 \\
\cdots & \cdots & \ddots & \ddots & \cdots \\
0 & 0 & 0 & \ddots & -Y \\
0 & 0 & 0 & \cdots & I_{n}
\end{array}\right] \in \mathbb{R}^{K n \times K n} .
$$

If $Z=\left[\begin{array}{ll}J_{K}(Y) S\end{array}\right] \in \mathbb{R}^{K n \times(K n+m)}$, where $S=\left[\begin{array}{c}S_{1} \\ S_{2} \\ \vdots \\ S_{K-1} \\ S_{K}\end{array}\right] \in \mathbb{R}^{K n \times m}$ with $S_{i} \in \mathbb{R}^{n \times n}$ $(i=1, \ldots, k)$, then

$$
Z^{\perp}=\operatorname{col}\left\{-\sum_{i=1}^{K} Y^{i-1} S_{i},-\sum_{i=2}^{K} Y^{i-2} S_{i}, \ldots,-S_{K}, I_{m}\right\} .
$$

Lemma 2 [27] (Finsler's Lemma) Consider real matrices B and M such that B has full row rank and $M=M^{T}$. The following statements are equivalent:

1. There exists a vector $x$ such that $x^{T} M x<0$ and $B x=0$;

2. There exists a scalar $l \in \mathbb{R}$ such that

$$
l B^{T} B-M>0
$$

3. The following condition holds:

$$
B^{\perp T} M B^{\perp}<0 .
$$




\section{Main Results}

\subsection{Stability}

If $A\left(r_{t}\right)$ and $A_{d}\left(r_{t}\right)$ are known and do not have polytopic uncertainties, then system $\Sigma_{p}$ becomes

$$
\begin{aligned}
\Sigma: \quad x(t) & =A\left(r_{t}\right) x(t)+A_{d}\left(r_{t}\right) x\left(t-\sum_{k=1}^{r} d_{k}\left(r_{t}\right)\right), \\
x(t) & =\varphi(t), \quad t \in[-\mu, 0],
\end{aligned}
$$

where for each possible value $r_{t}=i, i \in \tilde{N}$, hold $A_{i}=A(i)$ and $A_{d i}=A_{d i}(i)$. Define

$$
\eta=\max _{i \in \tilde{N}}\left\{\left|\pi_{i i}\right|\right\}, \quad \lambda_{i p}=\sum_{k=1}^{p} d_{i k}, \quad \bar{\lambda}_{i p}=\sum_{k=1}^{p} \bar{d}_{i k}, \quad i \in \tilde{N},
$$

with $\lambda_{i 0}=0$ and $\bar{\lambda}_{i 0}=0$, which leads to

$$
\lambda_{i p} \leq \bar{\lambda}_{i p} \leq \mu, \quad i \in \tilde{N}, l=0,1, \ldots, r .
$$

Then we have the following theorem by applying the methodology used in [13].

Theorem 1 If there exist $P_{i}>0, i \in \tilde{N}, Q_{m}>0, m=1, \ldots, r, X>0$ and $Y>0$ such that

$$
\begin{aligned}
& Q_{m+1}-Q_{m} \leq 0, \quad m=1, \ldots, r-1, \\
& T^{\perp T}\left[\begin{array}{ccc}
\Gamma_{i 1}+\Gamma_{i 2} & 0 & 0 \\
0 & \Gamma_{i 3} & 0 \\
0 & 0 & \Gamma_{i 4}
\end{array}\right] T^{\perp}<0,
\end{aligned}
$$

for each $i \in \tilde{N}$, then the system $\Sigma$ is stochastically stable, where

$$
\begin{aligned}
& \Gamma_{i 1}=\left[\begin{array}{ccccc}
\sum_{j=1}^{N} \pi_{i j} P_{j}+X+(1+\eta \mu) Q_{1} & 0 & \ldots & 0 & P_{i} A_{d i} \\
+P_{i} A_{i}+A_{i}^{T} P_{i} & Q_{2}-Q_{1} & \ldots & 0 & 0 \\
0 & \vdots & \ddots & \vdots & \vdots \\
\vdots & & & Q_{r}-Q_{r-1} & 0 \\
0 & 0 & & 0 & -Q_{r}
\end{array}\right] \\
& \in \mathbb{R}^{(r+1) n \times(r+1) n}, \\
& \Gamma_{i 2}=\left[\begin{array}{lllll}
A_{i}^{T} & 0 & \cdots & 0 & A_{d i}^{T}
\end{array}\right] \mu Y\left[\begin{array}{c}
A_{i} \\
0 \\
\vdots \\
0 \\
A_{d i}
\end{array}\right] \in \mathbb{R}^{(r+1) n \times(r+1) n},
\end{aligned}
$$


$\Gamma_{i 3}=\left[\begin{array}{ccccc}-X & 0 & \cdots & 0 & 0 \\ 0 & -\bar{d}_{i 1}^{-1} Y & \cdots & 0 & 0 \\ \vdots & \vdots & \ddots & \vdots & \vdots \\ 0 & 0 & \cdots & -\bar{d}_{i(r-1)}^{-1} Y & 0 \\ 0 & 0 & \cdots & 0 & -\left(\mu-\lambda_{i(r-1)}\right)^{-1} Y\end{array}\right]$

$\in \mathbb{R}^{(r+1) n \times(r+1) n}$,

$\Gamma_{i 4}=\left[\begin{array}{cccc}\pi_{i i} d_{i 1}^{-1} Q_{1} & \cdots & 0 & 0 \\ \vdots & \ddots & \vdots & \vdots \\ 0 & \cdots & \pi_{i i} d_{i(r-1)}^{-1} Q_{r-1} & 0 \\ 0 & \cdots & 0 & \pi_{i i} d_{i r}^{-1} Q_{r}\end{array}\right] \in \mathbb{R}^{r n \times r n}$

$T^{\perp}=\mathrm{col}\left\{-\sum_{i=1}^{r} S_{i},-\sum_{i=2}^{r} S_{i}, \ldots,-S_{r}, I_{(2 r+2) n}\right\} \in \mathbb{R}^{(3 r+2) n \times 2(r+1) n}$,

$\left[\begin{array}{c}S_{1} \\ S_{2} \\ \vdots \\ S_{r-1} \\ S_{r}\end{array}\right]=\left[\begin{array}{cccccc}0 & -I_{n} & 0 & \cdots & 0 & 0 \\ 0 & 0 & -I_{n} & \cdots & 0 & 0 \\ \vdots & \vdots & \vdots & \ddots & \vdots & \vdots \\ 0 & 0 & 0 & \cdots & -I_{n} & 0 \\ -I_{n} & 0 & 0 & \cdots & 0 & -I_{n}\end{array}\right]$.

Proof Note that $\left\{\left(x(t), r_{t}\right), t \geq 0\right\}$ is not a Markov process. From [4], we define a new process $\left\{\left(x_{t}, r_{t}\right), t \geq 0\right\}$ by

$$
x_{t}(s)=x(t+s), \quad t-\mu \leq s \leq t,
$$

which leads to conclusion that $\left\{\left(x_{t}, r_{t}\right), t \geq 0\right\}$ is a Markov process with initial state $\left(\varphi(\cdot), r_{0}\right)$. Define a new stochastic Lyapunov functional candidate of the system $\Sigma$ as

$$
V\left(x_{t}, r_{t}\right)=\sum_{p=1}^{5} V_{p}\left(x_{t}, r_{t}\right)
$$

where

$$
\begin{aligned}
& V_{1}\left(x_{t}, r_{t}\right)=x^{T}(t) P\left(r_{t}\right) x(t), \quad V_{2}\left(x_{t}, r_{t}\right)=\int_{t-\mu}^{t} x^{T}(s) X x(s) d s \\
& V_{3}\left(x_{t}, r_{t}\right)=\int_{-\mu}^{0} \int_{t+\theta}^{t} \dot{x}^{T}(s) Y \dot{x}(s) d s d \theta \\
& V_{4}\left(x_{t}, r_{t}\right)=\sum_{l=1}^{r} \int_{t-\lambda_{i l}}^{t-\lambda_{i(l-1)}} x^{T}(s) Q_{l} x(s) d s \\
& V_{5}\left(x_{t}, r_{t}\right)=\eta \int_{-\mu}^{0} \int_{t+\theta}^{t} x^{T}(s) Q_{1} x(s) d s d \theta .
\end{aligned}
$$


Let $\mathcal{A}$ denote the weak infinitesimal generator of the random process $\left\{x_{t}, r_{t}\right\}$. Then, for each $r_{t}=i, i \in \tilde{N}$, it can be verified that

$$
\begin{aligned}
& \mathcal{A}\left[V_{1}\left(x_{t}, i\right)\right]=x^{T}(t)\left(\sum_{j=1}^{N} \pi_{i j} P_{j}\right) x(t)+2 x^{T}(t) P_{i}\left(A_{i} x(t)+A_{d i} x\left(t-\lambda_{i r}\right)\right), \\
& \mathcal{A}\left[V_{2}\left(x_{t}, i\right)\right]=x^{T}(t) X x(t)-x^{T}(t-\mu) X x(t-\mu) .
\end{aligned}
$$

By Jensen's integral inequality and $0 \leq \lambda_{i(r-1)} \leq \mu, i \in \tilde{N}$, we have

$$
\begin{aligned}
& \mathcal{A}\left[V_{3}\left(x_{t}, i\right)\right]=\mu \dot{x}^{T}(t) Y \dot{x}(t)-\int_{t-\mu}^{t} \dot{x}^{T}(s) Y \dot{x}(s) d s \\
& =\mu \dot{x}^{T}(t) Y \dot{x}(t)-\int_{t-\mu}^{t-\lambda_{i(r-1)}} \dot{x}^{T}(s) Y \dot{x}(s) d s \\
& -\sum_{l=1}^{r-1} \int_{t-\lambda_{i l}}^{t-\lambda_{i(l-1)}} \dot{x}^{T}(s) Y \dot{x}(s) d s \\
& \leq \mu \dot{x}^{T}(t) Y \dot{x}(t)-\left(\mu-\lambda_{i(r-1)}\right)^{-1}\left(\int_{t-\mu}^{t-\lambda_{i(r-1)}} \dot{x}(s) d s\right)^{T} Y \\
& \times\left(\int_{t-\mu}^{t-\lambda_{i(r-1)}} \dot{x}(s) d s\right) \\
& -\sum_{l=1}^{r-1} d_{i l}^{-1}\left(\int_{t-\lambda_{i l}}^{t-\lambda_{i(l-1)}} \dot{x}(s) d s\right)^{T} Y\left(\int_{t-\lambda_{i l}}^{t-\lambda_{i(l-1)}} \dot{x}(s) d s\right) \\
& \leq \mu \dot{x}^{T}(t) Y \dot{x}(t)-\left(\mu-\lambda_{i(r-1)}\right)^{-1}\left(\int_{t-\mu}^{t-\lambda_{i(l-1)}} \dot{x}(s) d s\right)^{T} Y \\
& \times\left(\int_{t-\mu}^{t-\lambda_{i(l-1)}} \dot{x}(s) d s\right) \\
& -\sum_{l=1}^{r-1} \bar{d}_{i l}^{-1}\left(\int_{t-\lambda_{i l}}^{t-\lambda_{i(l-1)}} \dot{x}(s) d s\right)^{T} Y\left(\int_{t-\lambda_{i l}}^{t-\lambda_{i(l-1)}} \dot{x}(s) d s\right) .
\end{aligned}
$$

From the proof of Theorem 3.1 in [3] and

$$
V_{4}\left(x_{t}, i\right)=\sum_{l=1}^{r}\left(\int_{t-\lambda_{i l}}^{t} x^{T}(s) Q_{l} x(s) d s-\int_{t-\lambda_{i(l-1)}}^{t} x^{T}(s) Q_{l} x(s) d s\right),
$$

we have

$$
\mathbb{E}\left[V_{4}\left(x_{t+\Delta}, r_{t+\Delta}\right) \mid\left(x_{t}, r_{t}=i\right)\right]
$$




$$
\begin{aligned}
= & \sum_{j \neq i} \mathbb{E}\left[I _ { ( r _ { t + \Delta } = j ) } \left(\sum _ { l = 1 } ^ { r } \left(\int_{t}^{t+\Delta} x^{T}(s) Q_{l} x(s) d s\right.\right.\right. \\
& \left.\left.\left.+\int_{t+\Delta-\lambda_{j l}}^{t} x^{T}(s) Q_{l} x(s) d s\right)\right) \mid\left(x_{t}, r_{t}=i\right)\right] \\
& +\mathbb{E}\left[I_{\left(r_{t+\Delta}=i\right)}\left(\sum_{l=1}^{r}\left(\int_{t+\Delta-\lambda_{i l}}^{t+\Delta} x^{T}(s) Q_{l} x(s) d s\right)\right) \mid\left(x_{t}, r_{t}=i\right)\right] \\
& -\sum_{j \neq i} \mathbb{E}\left[I _ { ( r _ { t + \Delta } = j ) } \left(\sum _ { l = 1 } ^ { r } \left(\int_{t}^{t+\Delta} x^{T}(s) Q_{l} x(s) d s\right.\right.\right. \\
& \left.\left.\left.+\int_{t+\Delta-\lambda_{j(l-1)}}^{t} x^{T}(s) Q_{l} x(s) d s\right)\right) \mid\left(x_{t}, r_{t}=i\right)\right] \\
& -\mathbb{E}\left[I_{\left(r_{t+\Delta}=i\right)}\left(\sum_{l=1}^{r}\left(\int_{t+\Delta-\lambda_{i(l-1)}}^{t+\Delta} x^{T}(s) Q_{l} x(s) d s\right)\right) \mid\left(x_{t}, r_{t}=i\right)\right] \\
= & W_{1}-W_{2},
\end{aligned}
$$

where

$$
\begin{aligned}
W_{1}= & \sum_{j \neq i} \mathbb{E}\left[I _ { ( r _ { t + \Delta } = j ) } \left(\sum _ { l = 1 } ^ { r } \left(\int_{t}^{t+\Delta} x^{T}(s) Q_{l} x(s) d s\right.\right.\right. \\
& \left.\left.\left.+\int_{t+\Delta-\lambda_{j l}}^{t} x^{T}(s) Q_{l} x(s) d s\right)\right) \mid\left(x_{t}, r_{t}=i\right)\right] \\
& +\mathbb{E}\left[I_{\left(r_{t+\Delta}=i\right)}\left(\sum_{l=1}^{r}\left(\int_{t+\Delta-\lambda_{i l}}^{t+\Delta} x^{T}(s) Q_{l} x(s) d s\right)\right) \mid\left(x_{t}, r_{t}=i\right)\right] \\
W_{2}= & \sum_{j \neq i} \mathbb{E}\left[I _ { ( r _ { t + \Delta } = j ) } \sum _ { l = 1 } ^ { r } \left(\int_{t}^{t+\Delta} x^{T}(s) Q_{l} x(s) d s\right.\right. \\
& \left.\left.+\int_{t+\Delta-\lambda_{j(l-1)}}^{t} x^{T}(s) Q_{l} x(s) d s\right) \mid\left(x_{t}, r_{t}=i\right)\right] \\
& +\mathbb{E}\left[I_{\left(r_{t+\Delta}=i\right)}\left(\sum_{l=1}^{r}\left(\int_{t+\Delta-\lambda_{i(l-1)}}^{t+\Delta} x^{T}(s) Q_{l} x(s) d s\right)\right) \mid\left(x_{t}, r_{t}=i\right)\right] .
\end{aligned}
$$

The first part can be expressed as

$$
W_{1}=\sum_{j \neq i} \mathbb{E}\left[I _ { ( r _ { t + \Delta } = j ) } \left(\sum _ { l = 1 } ^ { r } \left(\int_{t}^{t+\Delta} x^{T}(s) Q_{l} x(s) d s\right.\right.\right.
$$




$$
\begin{aligned}
& \left.\left.\left.+\int_{t+\Delta-\lambda_{j l}}^{t} x^{T}(s) Q_{l} x(s) d s\right)\right) \mid\left(x_{t}, r_{t}=i\right)\right] \\
& +\mathbb{E}\left[I_{\left(r_{t+\Delta}=i\right)}\left(\sum_{l=1}^{r}\left(\int_{t+\Delta-\lambda_{i l}}^{t+\Delta} x^{T}(s) Q_{l} x(s) d s\right)\right) \mid\left(x_{t}, r_{t}=i\right)\right] \\
& =\mathcal{F}+\mathcal{F} \mathcal{F}+\mathcal{F} \mathcal{F} \mathcal{F},
\end{aligned}
$$

where

$$
\begin{aligned}
\mathcal{F} & =\sum_{j \neq i} \mathbb{E}\left[I_{\left(r_{t+\Delta}=j\right)}\left(\sum_{l=1}^{r} \int_{t}^{t+\Delta} x^{T}(s) Q_{l} x(s) d s\right) \mid\left(x_{t}, r_{t}=i\right)\right], \\
\mathcal{F} \mathcal{F} & =\sum_{j \neq i} \mathbb{E}\left[I_{\left(r_{t+\Delta}=j\right)}\left(\sum_{l=1}^{r} \int_{t+\Delta-\lambda_{j l}}^{t} x^{T}(s) Q_{l} x(s) d s\right) \mid\left(x_{t}, r_{t}=i\right)\right], \\
\mathcal{F} \mathcal{F} \mathcal{F} & =\mathbb{E}\left[I_{\left(r_{t+\Delta}=i\right)}\left(\sum_{l=1}^{r}\left(\int_{t+\Delta-\lambda_{i l}}^{t+\Delta} x^{T}(s) Q_{l} x(s) d s\right)\right) \mid\left(x_{t}, r_{t}=i\right)\right] .
\end{aligned}
$$

Note that

$$
\begin{aligned}
0 & \leq \mathcal{F} \leq o\left(\Delta^{2}\right) \\
\mathcal{F} \mathcal{F} & =\sum_{j \neq i} P\left[r_{t+\Delta}=j \mid r_{t}=i\right] \sum_{l=1}^{r}\left(\int_{t+\Delta-\lambda_{j l}}^{t} x^{T}(s) Q_{l} x(s) d s\right) \\
& =\sum_{j \neq i}\left(\pi_{i j} \Delta+o(\Delta)\right) \sum_{l=1}^{r}\left(\int_{t+\Delta-\lambda_{j l}}^{t} x^{T}(s) Q_{l} x(s) d s\right) \\
\mathcal{F} \mathcal{F} \mathcal{F} & =P\left[r_{t+\Delta}=i \mid r_{t}=i\right] \times \mathbb{E}\left[\sum_{l=1}^{r}\left(\int_{t+\Delta-\lambda_{i l}}^{t+\Delta} x^{T}(s) Q_{l} x(s) d s\right) \mid\left(x_{t}, r_{t}=i\right)\right] \\
& =\left(1+\pi_{i i} \Delta+o(\Delta)\right) \sum_{l=1}^{r}\left(\int_{t+\Delta-\lambda_{i l}}^{t+\Delta} x^{T}(s) Q_{l} x(s) d s\right) .
\end{aligned}
$$

Similarly,

$$
\begin{aligned}
W_{2}= & \sum_{j \neq i} \mathbb{E}\left[I_{\left(r_{t+\Delta}=j\right)}\left(\sum_{l=1}^{r}\left(\int_{t}^{t+\Delta} x^{T}(s) Q_{l} x(s) d s\right)\right) \mid\left(x_{t}, r_{t}=i\right)\right] \\
& +\sum_{j \neq i}\left(\pi_{i j} \Delta+o(\Delta)\right) \sum_{l=1}^{r}\left(\int_{t+\Delta-\lambda_{j(l-1)}}^{t} x^{T}(s) Q_{l} x(s) d s\right) \\
& +\left(1+\pi_{i i} \Delta+o(\Delta)\right) \sum_{l=1}^{r}\left(\int_{t+\Delta-\lambda_{j(l-1)}}^{t+\Delta} x^{T}(s) Q_{l} x(s) d s\right) .
\end{aligned}
$$




\section{Thus we have}

$$
\begin{aligned}
\left(\frac{1}{\Delta}\right)\left\{\mathbb{E}\left[V_{4}\left(x_{t+\Delta}, r_{t+\Delta}\right) \mid\left(x_{t}, r_{t}=i\right)\right]-V_{4}\left(x_{t}, r_{t}=i\right)\right\} \\
=\left(\frac{1}{\Delta}\right)\left(\sum_{l=1}^{r}\left(\int_{t+\Delta-\lambda_{i l}}^{t+\Delta} x^{T}(s) Q_{l} x(s) d s\right)-\sum_{l=1}^{r}\left(\int_{t-\lambda_{i l}}^{t} x^{T}(s) Q_{l} x(s) d s\right)\right) \\
+\pi_{i i} \sum_{l=1}^{r}\left(\int_{t+\Delta-\lambda_{i l}}^{t+\Delta} x^{T}(s) Q_{l} x(s) d s\right) \\
+\sum_{j \neq i} \pi_{i j} \sum_{l=1}^{r}\left(\int_{t+\Delta-\lambda_{j l}}^{t} x^{T}(s) Q_{l} x(s) d s\right) \\
\quad-\left(\frac{1}{\Delta}\right)\left(\sum_{l=1}^{r}\left(\int_{t+\Delta-\lambda_{i l}}^{t+\Delta} x^{T}(s) Q_{l} x(s) d s\right)-\sum_{l=1}^{r}\left(\int_{t-\lambda_{i l}}^{t} x^{T}(s) Q_{l} x(s) d s\right)\right) \\
\quad-\pi_{i i} \sum_{l=1}^{r}\left(\int_{t+\Delta-\lambda_{j(l-1)}}^{t+\Delta} x^{T}(s) Q_{l} x(s) d s\right) \\
\quad-\sum_{j \neq i} \pi_{i j} \sum_{l=1}^{r}\left(\int_{t+\Delta-\lambda_{j(l-1)}}^{t} x^{T}(s) Q_{l} x(s) d s\right) .
\end{aligned}
$$

Therefore, we obtain

$$
\begin{aligned}
& \mathcal{A}\left[V_{4}\left(x_{t}, i\right)\right]=\sum_{l=1}^{r} \mathcal{A}\left[\int_{t-\lambda_{i l}}^{t-\lambda_{i(l-1)}} x^{T}(s) Q_{l} x(s) d s\right] \\
& =\lim _{\Delta \rightarrow 0} \frac{1}{\Delta}\left\{\mathbb{E}\left[V_{4}\left(x_{t+\Delta}, r_{t+\Delta}\right) \mid\left(x_{t}, r_{t}=i\right)\right]-V_{4}\left(x_{t}, r_{t}=i\right)\right\} \\
& =\sum_{l=1}^{r}\left(\mathcal{A}\left[\int_{t-\lambda_{i l}}^{t} x^{T}(s) Q_{l} x(s) d s\right]-\mathcal{A}\left[\int_{t-\lambda_{i(l-1)}}^{t} x^{T}(s) Q_{l} x(s) d s\right]\right) \\
& =\sum_{l=1}^{r}\left(\begin{array}{c}
x^{T}(t) Q_{l} x(t)-x^{T}\left(t-\lambda_{i l}\right) Q_{l} x\left(t-\lambda_{i l}\right) \\
+\sum_{j=1}^{N} \pi_{i j} \int_{t-\lambda_{i l}}^{t} x^{T}(s) Q_{l} x(s) d s \\
-x^{T}(t) Q_{l} x(t)+x^{T}\left(t-\lambda_{i(l-1)}\right) Q_{l} x\left(t-\lambda_{i(l-1)}\right) \\
-\sum_{j=1}^{N} \pi_{i j} \int_{t-\lambda_{i l}}^{t} x^{T}(s) Q_{l} x(s) d s
\end{array}\right) \\
& =\sum_{l=1}^{r}\left(x^{T}\left(t-\lambda_{i(l-1)}\right) Q_{l} x\left(t-\lambda_{i(l-1)}\right)-x^{T}\left(t-\lambda_{i l}\right) Q_{l} x\left(t-\lambda_{i l}\right)\right) \\
& +\sum_{j=1}^{N} \pi_{i j} \sum_{l=1}^{r} \int_{t-\lambda_{j l}}^{t-\lambda_{j(l-1)}} x^{T}(s) Q_{l} x(s) d s .
\end{aligned}
$$


From (5) and (6) it can be further shown that

$$
\begin{aligned}
& \mathcal{A}\left[V_{4}\left(x_{t}, i\right)\right]=x^{T}(t) Q_{1} x(t)-x^{T}\left(t-\lambda_{i r}\right) Q_{r} x\left(t-\lambda_{i r}\right) \\
& -\sum_{l=1}^{r-1} x^{T}\left(t-\lambda_{i l}\right)\left(Q_{l}-Q_{l+1}\right) x\left(t-\lambda_{i l}\right) \\
& +\sum_{j=1, j \neq i}^{N} \pi_{i j} \sum_{l=1}^{r} \int_{t-\lambda_{j l}}^{t-\lambda_{j(l-1)}} x^{T}(s) Q_{l} x(s) d s \\
& -\left|\pi_{i i}\right| \sum_{l=1}^{r} \int_{t-\lambda_{i l}}^{t-\lambda_{i(l-1)}} x^{T}(s) Q_{l} x(s) d s \\
& \leq x^{T}(t) Q_{1} x(t)-x^{T}\left(t-\lambda_{i r}\right) Q_{r} x\left(t-\lambda_{i r}\right) \\
& -\sum_{l=1}^{r-1} x^{T}\left(t-\lambda_{i l}\right)\left(Q_{l}-Q_{l+1}\right) x\left(t-\lambda_{i l}\right) \\
& +\sum_{j=1, j \neq i}^{N} \pi_{i j} \sum_{l=1}^{r} \int_{t-\lambda_{j l}}^{t-\lambda_{j(l-1)}} x^{T}(s) Q_{l} x(s) d s \\
& -\left|\pi_{i i}\right| \sum_{l=1}^{r} d_{i l}^{-1} \int_{t-\lambda_{i l}}^{t-\lambda_{i(l-1)}} x^{T}(s) d s Q_{l} \int_{t-\lambda_{i l}}^{t-\lambda_{i(l-1)}} x(s) d s \\
& \leq x^{T}(t) Q_{1} x(t)-x^{T}\left(t-\lambda_{i r}\right) Q_{r} x\left(t-\lambda_{i r}\right) \\
& -\sum_{l=1}^{r-1} x^{T}\left(t-\lambda_{i l}\right)\left(Q_{l}-Q_{l+1}\right) x\left(t-\lambda_{i l}\right) \\
& +\sum_{j=1, j \neq i}^{N} \pi_{i j} \sum_{l=1}^{r} \int_{t-\lambda_{j l}}^{t-\lambda_{j(l-1)}} x^{T}(s) Q_{1} x(s) d s \\
& -\left|\pi_{i i}\right| \sum_{l=1}^{r} d_{i l}^{-1} \int_{t-\lambda_{i l}}^{t-\lambda_{i(l-1)}} x^{T}(s) d s Q_{l} \int_{t-\lambda_{i l}}^{t-\lambda_{i(l-1)}} x(s) d s \\
& \leq x^{T}(t) Q_{1} x(t)-x^{T}\left(t-\lambda_{i r}\right) Q_{r} x\left(t-\lambda_{i r}\right) \\
& -\sum_{l=1}^{r-1} x^{T}\left(t-\lambda_{i l}\right)\left(Q_{l}-Q_{l+1}\right) x\left(t-\lambda_{i l}\right) \\
& +\sum_{j=1, j \neq i}^{N} \pi_{i j} \int_{t-\mu}^{t} x^{T}(s) Q_{1} x(s) d s
\end{aligned}
$$




$$
\begin{aligned}
& -\left|\pi_{i i}\right| \sum_{l=1}^{r} d_{i l}^{-1} \int_{t-\lambda_{i l}}^{t-\lambda_{i(l-1)}} x^{T}(s) d s Q_{l} \int_{t-\lambda_{i l}}^{t-\lambda_{i(l-1)}} x(s) d s \\
= & x^{T}(t) Q_{1} x(t)-x^{T}\left(t-\lambda_{i r}\right) Q_{r} x\left(t-\lambda_{i r}\right) \\
& -\sum_{l=1}^{r-1} x^{T}\left(t-\lambda_{i l}\right)\left(Q_{l}-Q_{l+1}\right) x\left(t-\lambda_{i l}\right) \\
& -\pi_{i i} \int_{t-\mu}^{t} x^{T}(s) Q_{1} x(s) d s \\
& +\pi_{i i} d_{i l}^{-1} \int_{t-\lambda_{i l}}^{t-\lambda_{i(l-1)}} x^{T}(s) d s Q_{l} \int_{t-\lambda_{i l}}^{t-\lambda_{i(l-1)}} x(s) d s \\
\leq & x^{T}(t) Q_{1} x(t)-x^{T}\left(t-\lambda_{i r}\right) Q_{r} x\left(t-\lambda_{i r}\right) \\
& -\sum_{l=1}^{r-1} x^{T}\left(t-\lambda_{i l}\right)\left(Q_{l}-Q_{l+1}\right) x\left(t-\lambda_{i l}\right) \\
& +\eta \int_{t-\mu}^{t} x^{T}(s) Q_{1} x(s) d s \\
& +\pi_{i i} \sum_{l=1}^{r} d_{i l}^{-1} \int_{t-\lambda_{i l}}^{t-\lambda_{i(l-1)}} x^{T}(s) d s Q_{l}^{t-\lambda_{i(l-1)}} x(s) d s,
\end{aligned}
$$

and

$$
\mathcal{A}\left[V_{5}\left(x_{t}, i\right)\right]=\eta \mu x^{T}(t) Q_{1} x(t)-\eta \int_{t-\mu}^{t} x^{T}(s) Q_{1} x(s) d s .
$$

Then we have

$$
\begin{aligned}
\mathcal{A}\left[V\left(x_{t}, i\right)\right]= & \sum_{p=1}^{5} \mathcal{A}\left[V_{p}\left(x_{t}, i\right)\right] \\
\leq & x^{T}(t)\left(\sum_{j=1}^{N} \pi_{i j} P_{j}\right) x(t)+2 x^{T}(t) P_{i}\left(A_{i} x(t)+A_{d i} x\left(t-\lambda_{i r}\right)\right) \\
& +x^{T}(t) X x(t)-x^{T}(t-\mu) X x(t-\mu) \\
& +\mu \dot{x}^{T}(t) Y \dot{x}(t)-\left(\mu-\lambda_{i(r-1)}\right)^{-1}\left(\int_{t-\mu}^{t-\lambda_{i(r-1)}} \dot{x}(s) d s\right)^{T} Y \\
& \times\left(\int_{t-\mu}^{t-\lambda_{i(r-1)}} \dot{x}(s) d s\right) \\
& -\sum_{l=1}^{r-1}\left(\int_{t-\lambda_{i l}}^{t-\lambda_{i(l-1)}} \dot{x}(s) d s\right)^{T}\left(\bar{d}_{i l}^{-1} Y\right)\left(\int_{t-\lambda_{i l}}^{t-\lambda_{i(l-1)}} \dot{x}(s) d s\right)
\end{aligned}
$$




$$
\begin{aligned}
& +x^{T}(t) Q_{1} x(t)-x^{T}\left(t-\lambda_{i r}\right) Q_{r} x\left(t-\lambda_{i r}\right) \\
& +\eta \mu x^{T}(t) Q_{1} x(t)-\sum_{l=1}^{r-1} x^{T}\left(t-\lambda_{i l}\right)\left(Q_{l}-Q_{l+1}\right) x\left(t-\lambda_{i l}\right) \\
& +\pi_{i i} \sum_{l=1}^{r} d_{i l}^{-1}\left(\int_{t-\lambda_{i l}}^{t-\lambda_{i(l-1)}} x^{T}(s) d s\right) Q_{l}\left(\int_{t-\lambda_{i l}}^{t-\lambda_{i(l-1)}} x(s) d s\right),
\end{aligned}
$$

which equals

$$
\mathcal{A}\left[V\left(x_{t}, i\right)\right] \leq \zeta^{T}(t)\left[\begin{array}{ccc}
\Gamma_{i 1}+\Gamma_{i 2} & 0 & 0 \\
0 & \Gamma_{i 3} & 0 \\
0 & 0 & \Gamma_{i 4}
\end{array}\right] \zeta(t)
$$

where $\Gamma_{i j}, i \in \tilde{N}, j=1,2,3$, are defined in (8)-(11) and

$$
\begin{aligned}
\zeta(t)= & {\left[\begin{array}{lllll}
x(t) & x\left(t-\lambda_{i 1}\right) & \cdots & x\left(t-\lambda_{i r}\right)
\end{array}\right.} \\
& x(t-\mu) \quad \int_{t-\lambda_{i 1}}^{t} \dot{x}(s) d s \quad \cdots \quad \int_{t-\lambda_{i(r-1)}}^{t-\lambda_{i(r-2)}} \dot{x}(s) d s \quad \int_{t-\mu}^{t-\lambda_{i(r-1)}} \dot{x}(s) d s \\
& \left.\int_{t-\lambda_{i 1}}^{t} x(s) d s \quad \cdots \quad \int_{t-\lambda_{i(r-1)}}^{t-\lambda_{i(r-2)}} x(s) d s \quad \int_{t-\lambda_{i r}}^{t-\lambda_{i(r-1)}} x(s) d s\right]^{T}
\end{aligned}
$$

From the Newton-Leibniz formula we have

$$
\begin{aligned}
x(t)-x\left(t-\lambda_{i 1}\right)-\int_{t-\lambda_{i 1}}^{t} \dot{x}(s) d s & =0, \\
x\left(t-\lambda_{i 1}\right)-x\left(t-\lambda_{i 2}\right)-\int_{t-\lambda_{i 2}}^{t-\lambda_{i 1}} \dot{x}(s) d s & =0, \\
\vdots & \\
x\left(t-\lambda_{i(r-2)}\right)-x\left(t-\lambda_{i(r-1)}\right)-\int_{t-\lambda_{i(r-1)}}^{t-\lambda_{i(r-2)}} \dot{x}(s) d s & =0, \\
x\left(t-\lambda_{i(r-1)}\right)-x(t-\mu)-\int_{t-\mu}^{t-\lambda_{i(r-1)}} \dot{x}(s) d s & =0,
\end{aligned}
$$

which is equivalent to

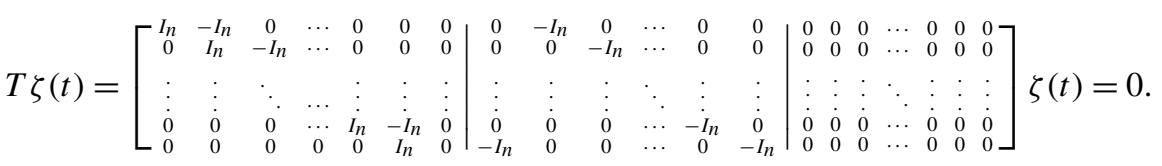


Therefore, from Lemma 2, $\mathcal{A}\left[V\left(x_{t}, i\right)\right]<0$ if

$$
\zeta^{T}(t)\left(T^{T} T-\left[\begin{array}{ccc}
\Gamma_{i 1}+\Gamma_{i 2} & 0 & 0 \\
0 & \Gamma_{i 3} & 0 \\
0 & 0 & \Gamma_{i 4}
\end{array}\right]\right) \zeta(t)>0,
$$

which is equivalent to (7) from Lemma 2, where $T^{\perp}$ can be computed from Lemma 1 and given in (12). This completes the proof of Theorem 1.

Remark 1 Sufficient condition with reduced conservatism is obtained for the stability analysis of Markovian jump system $\Sigma$ with mode-dependent multiple time delays. The conservatism can be reduced further by introducing more delay components as shown in Remarks 4, 5 and 6 in [13] at the expense of more matrix variables and LMIs in larger dimension in Theorem 1.

\subsection{Robust Stability}

Now we consider the robust stochastic stability of system $\Sigma_{p}$.

Theorem 2 If there exist $P_{i}>0, i \in \tilde{N}, Q_{m}>0, m=1, \ldots, r, X>0$ and $Y>0$ such that

$$
\begin{aligned}
& Q_{m+1}-Q_{m} \leq 0, \quad m=1, \ldots, r-1, \\
& T^{\perp T}\left[\begin{array}{ccc}
\Gamma_{i l 1}^{\prime}+\Gamma_{i l 2}^{\prime} & 0 & 0 \\
0 & \Gamma_{i 3} & 0 \\
0 & 0 & \Gamma_{i 4}
\end{array}\right] T^{\perp}<0,
\end{aligned}
$$

for each $i \in \tilde{N}, l=1,2, \ldots, q$, then the system $\Sigma_{p}$ is robust stochastically stable, where

$$
\begin{aligned}
& \Gamma_{i l 1}^{\prime}=\left[\begin{array}{ccccc}
\sum_{j=1}^{N} \pi_{i j} P_{j}+X+(1+\eta \mu) Q_{1} & 0 & \cdots & 0 & P_{i} A_{d i l} \\
+P_{i} A_{i l}+A_{i l}^{T} P_{i} & Q_{2}-Q_{1} & \cdots & 0 & 0 \\
0 & \vdots & \ddots & \vdots & \vdots \\
\vdots & & & Q_{r}-Q_{r-1} & 0 \\
0 & 0 & & 0 & -Q_{r}
\end{array}\right], \\
& \Gamma_{i l 2}^{\prime}=\left[\begin{array}{lllll}
A_{i l}^{T} & 0 & \cdots & 0 & A_{d i l}^{T}
\end{array}\right] \mu Y\left[\begin{array}{c}
A_{i l} \\
0 \\
\vdots \\
0 \\
A_{d i l}
\end{array}\right] \text {. }
\end{aligned}
$$

Proof From the proof of Theorem 1, it is easy to show that $\mathcal{A}\left[V\left(x_{t}, i\right)\right]$ in (13) satisfies

$$
\mathcal{A}\left[V\left(x_{t}, i\right)\right] \leq x^{T}(t)\left(\sum_{j=1}^{N} \pi_{i j} P_{j}+X+(1+\eta \mu) Q_{1}\right) x(t)
$$




$$
\begin{aligned}
& +2 x^{T}(t) P_{i}\left(A(i) x(t)+A_{d}(i) x\left(t-\lambda_{i r}\right)\right) \\
& -x^{T}(t-\mu) X x(t-\mu)-x^{T}\left(t-\lambda_{i r}\right) Q_{r} x\left(t-\lambda_{i r}\right) \\
& +\mu \dot{x}^{T}(t) Y \dot{x}(t)-\left(\mu-\lambda_{i(r-1)}\right)^{-1}\left(\int_{t-\mu}^{t-\lambda_{i(r-1)}} \dot{x}(s) d s\right)^{T} Y \\
& \times\left(\int_{t-\mu}^{t-\lambda_{i(r-1)}} \dot{x}(s) d s\right) \\
& -\sum_{l=1}^{r-1} \bar{d}_{i l}^{-1}\left(\int_{t-\lambda_{i l}}^{t-\lambda_{i(l-1)}} \dot{x}(s) d s\right)^{T} Y\left(\int_{t-\lambda_{i l}}^{t-\lambda_{i(l-1)}} \dot{x}(s) d s\right) \\
& -\sum_{l=1}^{r-1} x^{T}\left(t-\lambda_{i l}\right)\left(Q_{l}-Q_{l+1}\right) x\left(t-\lambda_{i l}\right) \\
& +\pi_{i i} \sum_{l=1}^{r} d_{i l}^{-1} \int_{t-\lambda_{i l}}^{t-\lambda_{i(l-1)}} x^{T}(s) d s Q_{l} \int_{t-\lambda_{i l}}^{t-\lambda_{i(l-1)}} x(s) d s \\
& =\zeta^{T}(t)\left[\begin{array}{ccc}
\tilde{\Gamma}_{i 1}+\tilde{\Gamma}_{i 2} & 0 & 0 \\
0 & \Gamma_{i 3} & 0 \\
0 & 0 & \Gamma_{i 4}
\end{array}\right] \zeta(t)
\end{aligned}
$$

where

$$
\begin{aligned}
& \tilde{\Gamma}_{i 1}=\left[\begin{array}{ccccc}
\sum_{j=1}^{N} \pi_{i j} P_{j}+X+(1+\eta \mu) Q_{1} & 0 & \cdots & 0 & P_{i} A_{d}(i) \\
+P_{i} A(i)+A^{T}(i) P_{i} & Q_{2}-Q_{1} & \cdots & 0 & 0 \\
0 & \vdots & \ddots & \vdots & \vdots \\
\vdots & & & Q_{r}-Q_{r-1} & 0 \\
0 & 0 & & 0 & -Q_{r}
\end{array}\right], \\
& \tilde{\Gamma}_{i 2}=\left[\begin{array}{lllll}
A^{T}(i) & 0 & \cdots & 0 & A_{d}^{T}(i)
\end{array}\right] \mu Y\left[\begin{array}{c}
A(i) \\
0 \\
\vdots \\
0 \\
A_{d}(i)
\end{array}\right]
\end{aligned}
$$

and $\zeta(t)$ is defined in (14), $\Gamma_{i 3}$ and $\Gamma_{i 4}$ are given in (10)-(11), and $A(i)$ and $A_{d}(i)$ are defined in (2). From the proof of Theorem 1,

$$
\zeta^{T}(t)\left[\begin{array}{ccc}
\tilde{\Gamma}_{i 1}+\tilde{\Gamma}_{i 2} & 0 & 0 \\
0 & \Gamma_{i 3} & 0 \\
0 & 0 & \Gamma_{i 4}
\end{array}\right] \zeta(t)<0
$$

if and only if

$$
T^{\perp T}\left[\begin{array}{ccc}
\tilde{\Gamma}_{i 1}+\tilde{\Gamma}_{i 2} & 0 & 0 \\
0 & \Gamma_{i 3} & 0 \\
0 & 0 & \Gamma_{i 4}
\end{array}\right] T^{\perp}<0,
$$


which, by Schur complement equivalence, is equivalent to

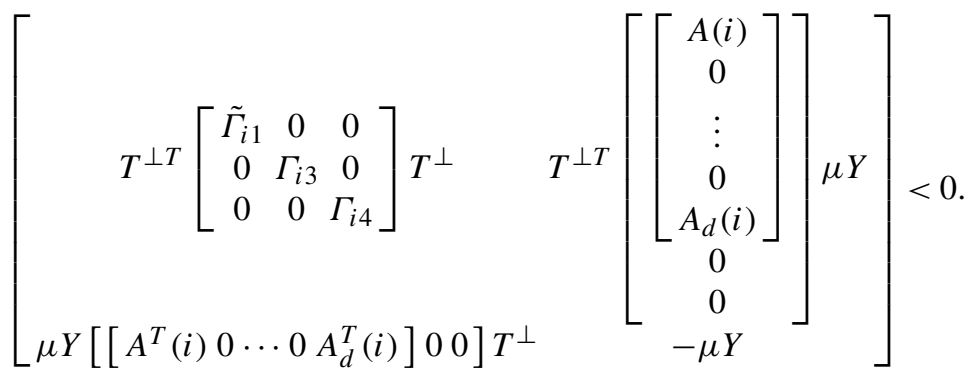

According to (16) and by Schur complement equivalence again, we have

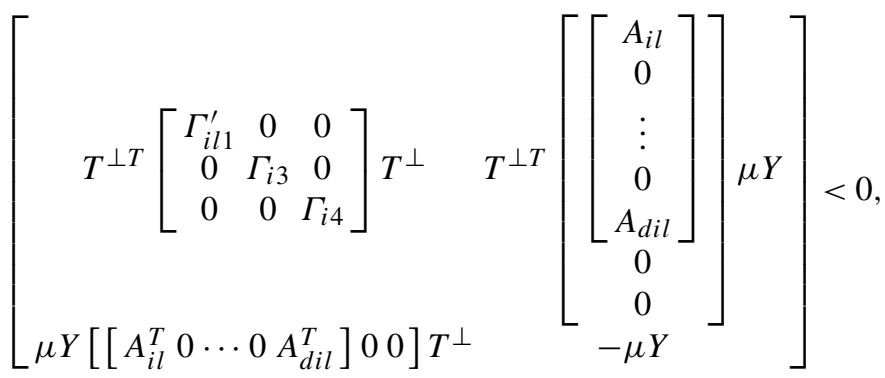

which further leads to

$$
\begin{aligned}
& \sum_{l=1}^{q} \alpha_{l}(t)\left[T^{\perp T}\left[\begin{array}{ccc}
\Gamma_{i l 1}^{\prime} & 0 & 0 \\
0 & \Gamma_{i 3} & 0 \\
0 & 0 & \Gamma_{i 4}
\end{array}\right] T^{\perp} T^{\perp T}\left[\begin{array}{c}
{\left[\begin{array}{c}
A_{i l} \\
0 \\
\vdots \\
0 \\
A_{d i l}
\end{array}\right]} \\
0 \\
0 \\
-\mu Y
\end{array}\right] \mu Y\right] \\
& \left.\left.\left.T^{\perp T}\left[\begin{array}{ccc}
\tilde{\Gamma}_{i 1} & 0 & 0 \\
0 & \Gamma_{i 3} & 0 \\
0 & 0 & \Gamma_{i 4}
\end{array}\right] T^{\perp} \quad T^{\perp T}\left[\begin{array}{c}
A(i) \\
0 \\
\vdots \\
0 \\
A_{d}(i) \\
0 \\
0 \\
-\mu Y
\end{array}\right] \mu A^{T}(i) 0 \cdots 0 A_{d}^{T}(i)\right] 00\right] T^{\perp}\right]<0
\end{aligned}
$$


By Schur complement equivalence again, it is easy to obtain that (18) holds if and only if (16) is satisfied, which implies

$$
\mathcal{A}\left[V\left(x_{t}, i\right)\right] \leq \zeta^{T}(t)\left[\begin{array}{ccc}
\tilde{\Gamma}_{i 1}+\tilde{\Gamma}_{i 2} & 0 & 0 \\
0 & \Gamma_{i 3} & 0 \\
0 & 0 & \Gamma_{i 4}
\end{array}\right] \zeta(t)<0 .
$$

This completes the proof of Theorem 2.

\section{Numerical Examples}

Next, two numerical examples will be given to illustrate the effectiveness of the proposed approach.

Example 1 The following matrices,

$$
\begin{array}{ll}
A_{11}=\left[\begin{array}{cc}
-2.2 & 0 \\
0 & 0.1
\end{array}\right], & A_{12}=\left[\begin{array}{cc}
-2 & 0 \\
0.2 & -1.6
\end{array}\right], \quad A_{21}=\left[\begin{array}{cc}
-2.1 & 0 \\
-0.1 & 0.9
\end{array}\right], \\
A_{22}=\left[\begin{array}{cc}
-1.4 & 0.1 \\
0.2 & -1.9
\end{array}\right], & A_{d 11}=\left[\begin{array}{cc}
-0.1 & 0 \\
-0.2 & -0.1
\end{array}\right], \quad A_{d 12}=\left[\begin{array}{cc}
-0.1 & 0 \\
0 & 0.2
\end{array}\right], \\
A_{d 21}=\left[\begin{array}{cc}
-0.2 & 0.1 \\
0.2 & 0
\end{array}\right], & A_{d 22}=\left[\begin{array}{cc}
0.1 & 0 \\
0.2 & 0.1
\end{array}\right],
\end{array}
$$

are the system matrices of a Markovian jump system $\Sigma_{p}$ with mode-dependent delays and polytopic uncertainties in the form of (1). The delays are given by

$$
d_{11}=0.8, \quad d_{12}=0.26, \quad d_{21}=0.26, \quad d_{22}=0.8,
$$

and the transition probability matrix is

$$
M=\left[\begin{array}{cc}
-1 & 1 \\
2 & -2
\end{array}\right]
$$

The solutions of (15) and (16) are given by

$$
\begin{aligned}
& X=\left[\begin{array}{cc}
114.2080 & 0.0058 \\
0.0058 & 0.0043
\end{array}\right]>0, \quad Y=\left[\begin{array}{cc}
655.4847 & 0.4929 \\
0.4929 & 0.0207
\end{array}\right]>0, \\
& P_{1}=10^{3} \times\left[\begin{array}{ll}
1.9823 & 0.0274 \\
0.0274 & 0.1872
\end{array}\right]>0, \quad P_{2}=10^{3} \times\left[\begin{array}{ll}
2.0173 & 0.0246 \\
0.0246 & 0.0836
\end{array}\right]>0, \\
& Q_{1}=10^{3} \times\left[\begin{array}{ll}
1.3046 & 0.0179 \\
0.0179 & 0.0104
\end{array}\right]>0, \quad Q_{2}=10^{3} \times\left[\begin{array}{ll}
1.2973 & 0.0179 \\
0.0179 & 0.0104
\end{array}\right]>0 .
\end{aligned}
$$

So the overall stability bound on delay is the sum of all parts, i.e. 1.06. 
Example 2 The system matrices of a Markovian jump system $\Sigma_{p}$ with modedependent delays and polytopic uncertainties in the form of (1) are given by

$$
\begin{aligned}
& A_{11}=\left[\begin{array}{cc}
-3 & 0 \\
0 & -3
\end{array}\right], \quad A_{12}=\left[\begin{array}{cc}
-3 & -0.1 \\
-0.1 & -4
\end{array}\right], \quad A_{21}=\left[\begin{array}{cc}
-2 & 0 \\
0 & -4
\end{array}\right] \text {, } \\
& A_{22}=\left[\begin{array}{cc}
-4 & 0.1 \\
0.1 & -3
\end{array}\right], \quad A_{d 11}=\left[\begin{array}{cc}
-1 & 0 \\
-1 & -1
\end{array}\right], \quad A_{d 12}=\left[\begin{array}{cc}
-0.8 & 0.1 \\
0.9 & 0
\end{array}\right] \text {, } \\
& A_{d 21}=\left[\begin{array}{cc}
-0.9 & 0 \\
0 & 0.7
\end{array}\right], \quad A_{d 22}=\left[\begin{array}{cc}
1 & 0 \\
0.9 & 0.6
\end{array}\right] \text {, }
\end{aligned}
$$

with

$$
d_{11}=1.22, \quad d_{12}=0.5, \quad d_{21}=1.21, \quad d_{22}=0.51 .
$$

The transition probability matrix is

$$
M=\left[\begin{array}{cc}
-3 & 3 \\
2 & -2
\end{array}\right] \text {. }
$$

The solutions of (15) and (16) are given by

$$
\begin{aligned}
& X=\left[\begin{array}{cc}
0.0306 & -0.0165 \\
-0.0165 & 0.0658
\end{array}\right]>0, \quad Y=\left[\begin{array}{cc}
0.3397 & 0.0958 \\
0.0958 & 0.0306
\end{array}\right]>0, \\
& P_{1}=\left[\begin{array}{cc}
17.3223 & 0.0878 \\
0.0878 & 0.5715
\end{array}\right]>0, \quad P_{2}=\left[\begin{array}{cc}
22.7518 & -0.0839 \\
-0.0839 & 0.4851
\end{array}\right]>0, \\
& Q_{1}=\left[\begin{array}{cc}
7.9497 & -0.0401 \\
-0.0401 & 0.3088
\end{array}\right]>0, \quad Q_{2}=\left[\begin{array}{cc}
7.9479 & -0.0380 \\
-0.0380 & 0.3019
\end{array}\right]>0 .
\end{aligned}
$$

So the overall stability bound on delay is the sum of all parts, i.e. 1.72. By the method in [4] without partitioning the delay, the upper bound of the time delay is 0.57 , which shows that our method has improved the result in [4].

\section{Conclusion}

We have presented solutions to the stability analysis for Markovian jump systems with multiple delay components and possible polytopic uncertainties. A delaydependent sufficient condition in terms of the LMI framework has been obtained by using a new form of stochastic Lyapunov functionals. Two examples have been given to demonstrate the effectiveness of the proposed result.

Open Access This article is distributed under the terms of the Creative Commons Attribution Noncommercial License which permits any noncommercial use, distribution, and reproduction in any medium, provided the original author(s) and source are credited. 


\section{References}

1. M. Basin, A. Rodkina, On delay-dependent stability for a class of nonlinear stochastic systems with multiple state delays. Nonlinear Anal. 68(8), 2147-2157 (2008)

2. E.K. Boukas, Z.K. Liu, Robust $H_{\infty}$ control of discrete-time Markovian jump linear systems with mode-dependent time-delays. IEEE Trans. Autom. Control 46(12), 1918-1924 (2001)

3. E.K. Boukas, Z.K. Liu, Robust stability and stabilizability of Markov jump linear uncertain systems with mode-dependent time-delays. J. Optim. Theory Appl. 109(3), 587-600 (2001)

4. E.K. Boukas, Z.K. Liu, G.X. Liu, Delay-dependent robust stability and $H_{\infty}$ control of jump linear systems with time-delay. Int. J. Control 74(4), 329-340 (2001)

5. E.K. Boukas, P. Shi, K. Benjelloun, On stabilization of uncertain linear systems with jump parameters. Int. J. Control 72(9), 842-850 (1999)

6. S. Boyd, L.E. Ghaoui, E. Feron, N. Balakrishnan, Linear Matrix Inequalities in System and Control Theory (Society for Industrial and Applied Mathematics, Philadelphia, 1994)

7. Y. Cao, J. Lam, Robust $H_{\infty}$ control of uncertain Markovian jump systems with time-delay. IEEE Trans. Autom. Control 45(1), 77-83 (2000)

8. W. Chen, J. Xu, Z. Guan, Guaranteed cost control for uncertain Markovian jump systems with modedependent time-delays. IEEE Trans. Autom. Control 48(12), 2270-2277 (2003)

9. O.L.V. Costa, J.B.R. do Val, J.C. Geromel, Continuous-time state-feedback $\mathrm{H}_{2}$-control of Markovian jump linear systems via convex analysis. Automatica 35(2), 259-268 (1999)

10. C.E. de Souza, Robust stability and stabilization of uncertain discrete-time Markovian jump linear systems. IEEE Trans. Autom. Control 51(5), 836-841 (2006)

11. C.E. de Souza, M.D. Fragoso, $H_{\infty}$ filtering for Markovian jump linear systems. Int. J. Syst. Sci. 33(11), 909-915 (2002)

12. C.E. de Souza, A. Trofino, K.A. Barbosa, Mode-independent $H_{\infty}$ filters for Markovian jump linear systems. IEEE Trans. Autom. Control 51(11), 1837-1841 (2006)

13. B. Du, J. Lam, Z. Shu, Z. Wang, A delay-partitioning projection approach to stability analysis of continuous systems with multiple delay components. IET Control Theory Appl. 3(4), 383-390 (2009)

14. H. Gao, T. Chen, J. Lam, A new delay system approach to network-based control. Automatica 44(1), 39-52 (2008)

15. H. Gao, J. Lam, S. Xu, C. Wang, Stabilization and $H_{\infty}$ control of two-dimensional Markovian jump systems. IMA J. Math. Control Inf. 21(4), 377-392 (2004)

16. Y. Ji, H.J. Chizeck, Controllability, observability and discrete-time Markovian jump linear quadratic control. Int. J. Control 48(2), 481-498 (1988)

17. Y. Ji, H.J. Chizeck, Jump linear quadratic Gaussian control: steady-state solution and testable conditions. Control Theory Adv. Technol. 6(3), 289-319 (1990)

18. Y. Ji, H.J. Chizeck, Jump linear quadratic Gaussian control in continuous time. IEEE Trans. Autom. Control 37(12), 1884-1892 (1992)

19. Y. Kang, J.F. Zhang, S.S. Ge, Robust output feedback $H_{\infty}$ control of uncertain Markovian jump systems with mode-dependent time-delays. Int. J. Control 81(1), 43-61 (2008)

20. J. Lam, H. Gao, C. Wang, Stability analysis for continuous systems with two additive time-varying delay components. Syst. Control Lett. 56(1), 16-24 (2007)

21. M. Liu, D.W.C. Ho, Y. Niu, Stabilization of Markovian jump linear system over networks with random communication delay. Automatica 45(2), 416-421 (2009)

22. S.K. Nguang, W. Assawinchaichote, P. Shi, $H_{\infty}$ filter for uncertain Markovian jump nonlinear systems: an LMI approach. Circuits Syst. Signal Process. 26(6), 853-874 (2007)

23. J. Qiu, H. Yang, P. Shi, Y. Xia, Robust $H_{\infty}$ control for class of discrete-time Markovian jump systems with time-varying delays based on delta operator. Circuits Syst. Signal Process. 27(5), 627-643 (2008)

24. A. Rodkina, M. Basin, On delay-dependent stability for a class of nonlinear stochastic delaydifferential equations. Math. Control Signals Syst. 18(2), 187-197 (2006)

25. A. Rodkina, M. Basin, On delay-dependent stability for vector nonlinear stochastic delay-difference equations with Volterra diffusion term. Syst. Control Lett. 6, 423-430 (2007)

26. H. Shao, Delay-range-dependent robust $H_{\infty}$ filtering for uncertain stochastic systems with modedependent time delays and Markovian jump parameters. J. Math. Anal. Appl. 342(2), 1084-1095 (2008)

27. R.E. Skelton, T. Iwasaki, K.M. Grigoriadis, A Unified Algebraic Approach to Linear Control Design (Taylor \& Francis Ltd., London, 1998) 
28. M.G. Todorov, M.D. Fragoso, Output feedback $H_{\infty}$ control of continuous-time infinite Markovian jump linear systems via LMI methods. SIAM J. Control Optim. 47(2), 950-974 (2008)

29. W.L. Tseng, C.Z. Chen, E. Gad, M. Nakhla, R. Achar, Passive order reduction for RLC circuits with delay elements. IEEE Trans. Adv. Packag. 30(4), 830-840 (2007)

30. Q. Wang, J. Lam, S. Xu, Q. Zhang, $H_{\infty}$ model approximation for discrete-time Markovian jump systems with mode-dependent time delays, in Proceedings of the 44th IEEE Conf. on Decis. and Control, and the Eur. Control Conf. (2005), pp. 6122-6127

31. Y. Wang, H. Zhang, $H_{\infty}$ control for uncertain Markovian jump systems with mode-dependent mixed delays. Prog. Nat. Sci. 18(3), 309-314 (2008)

32. Z. Wang, J. Lam, X. Liu, Nonlinear filtering for state delayed systems with Markovian switching. IEEE Trans. Signal Process. 51(9), 2321-2328 (2003)

33. Z. Wang, J. Lam, X. Liu, Exponential filtering for uncertain Markovian jump time-delay systems with nonlinear disturbances. IEEE Trans. Circuits Syst. II, Analog Digit. Signal Process. 51(5), 262-268 (2004)

34. J. Xia, B. Song, J. Lu, Robust $H_{\infty}$ control for stochastic time-delay systems with Markovian jump parameters via parameter-dependent Lyapunov functionals. Circuits Syst. Signal Process. 27(3), 331349 (2008)

35. J. Xiong, J. Lam, H. Gao, D.W.C. Ho, On robust stabilization of Markovian jump systems with uncertain switching probabilities. Automatica 41(5), 897-903 (2005)

36. S. Xu, T. Chen, J. Lam, Robust $H_{\infty}$ filtering for uncertain Markovian jump systems with modedependent time delays. IEEE Trans. Autom. Control 48(5), 900-907 (2003)

37. S. Xu, J. Lam, Robust Control and Filtering of Singular Systems, Lecture Notes in Control and Information Sciences, vol. 332 (Springer, Berlin, 2006)

38. S. Xu, J. Lam, A survey of linear matrix inequality techniques in stability analysis of delay systems. Int. J. Syst. Sci. 39(12), 1095-1113 (2008)

39. S. Xu, J. Lam, X. Mao, Delay-dependent $H_{\infty}$ control and filtering for uncertain Markovian jump systems with time-varying delays. IEEE Trans. Circuits Syst. I, Fundam. Theory Appl. 54(9), 20702077 (2007)

40. D. Yue, J. Fang, S. Won, Delay-dependent robust stability of stochastic uncertain systems with time delay and Markovian jump parameters. Circuits Syst. Signal Process. 22(4), 351-365 (2003)

41. B. Zhang, S. Xu, Y. Zou, Output feedback stabilization for delayed large-scale stochastic systems with Markovian jumping parameters. Asian J. Control 11(4), 457-460 (2009)

42. L. Zhang, B. Huang, J. Lam, $H_{\infty}$ model reduction of Markovian jump linear systems. Syst. Control Lett. 50(2), 103-118 (2003) 